\title{
Bridging the Gaps: Parental Involvement and Asian Non-native English Speakers' Postsecondary Enrollment
}

\author{
Ellen Yeh \\ Columbia College Chicago \\ USA \\ Guofang Wan \\ University of West Florida \\ USA
}

\begin{abstract}
While the number of the Asian non-native English speakers (NNESs) is increasing, their postsecondary education (PSE) enrollment rate has remained low in comparison to enrollment rates of Asian native-English speakers (NESs). The achievement gap in postsecondary enrollment between NNESs and NESs has widened, due not only to content areas such as reading and language arts that require higher English proficiency, but also sociocultural factors such as parental involvement. The current study investigates the extent to which parental involvement factors predict the likelihood of Asian NNESs' PSE enrollment after controlling for socioeconomic and linguistic factors. This study, an expansion of previous work exploring parental involvement and NNESs' PSE in the US, uses the national representative data from the Education Longitudinal Study dataset in 2002 (ELS: 2002) and a binary multilevel logistic regression model analysis. The results indicate that parental involvement is related to a greater likelihood of attending PSE institutions. Among the various forms of parental involvement, parent-student involvement and parent-school involvement were shown to have the greatest impact on Asian NNESs' PSE enrollment. The results also show that Asian NNESs' enrollment is higher if their parents participate in school volunteer work. Limitations, future studies, and implications for educators, parents and school policy makers are also discussed.
\end{abstract}

Keywords: asian non-native English speakers, asian students' post-secondary enrollment, parental involvement 


\section{Introduction}

Asian American students are regarded as a high-achieving population in higher education in the US, due to the unique style of Asian parenting (Seal, 2010). The 2000 US Census reported that $44 \%$ of Asian Americans received a bachelor's degree, which was much higher than the white student population (26\%) (Seal, 2010). In addition, Asian Americans have a higher rate of enrolling in selective four-year universities. In 2008, there were only $14 \%$ of the Asian American population in the state of California, but the university enrollment in fall 2010 showed that $40 \%$ of the total freshmen at UCLA were Asian Americans, and $37 \%$ of the freshmen in University of California, Berkeley were Asian Americans (Seal, 2010). However, it is hard to generalize Asian populations since they include diverse sociocultural and linguistic aspects, as well as different cultural values and norms. Although many Asian American families are regarded as the "model minority," educators shouldn't neglect the fact that many students are non-English native speakers (NNESs) whose parents are often ill-equipped to support NNESs in the complex and foreign US educational system (Gándara \& Rumberger, 2009; Her, 2014; Lee et al., 2017; Li \& Wang, 2012; Paik, Rahman, Kula, Saito, \& Witenstein, 2017; Suárez-Orozco, Pimentel, \& Martin, 2009). Prior research investigating the impacts of socioeconomic stress and low language proficiency levels on academic attainments has predominantly focused on European Americans and African Americans to the exclusion of adolescents from immigrant backgrounds and particularly those with Asian heritages (Kiang, Andrews, Stein, Supple, \& Gonzalez, 2013).

The purpose of this study is to investigate the impact of multiple parental involvement factors on postsecondary education (PSE) of Asian NNES students in the US. This study drew on the theoretical framework of sociocultural theory (Lantolf \& Thorne, 2006; Vygotsky, 1978, 1987) to explore the relationships among Asian NNESs, parents, schools, and communities. The study also adapted Epstein and Sanders' (2002) six concepts of parental involvement as criteria to select parental involvement factors and parental participation in students' lives in both home and school settings. Rather than regarding parental involvement as a single factor, parental involvement is categorized into four components: parent-school involvement, parent-student involvement, home culture, and home language environment. This study, being an expansion of previous work (Yeh, 2014), which explored parental involvement and PSE of all NNES populations in the US, uses the national representative data from the Education Longitudinal 
Study dataset in 2002 [ELS: 2002] (Ingels et al., 2007; National Center for Education Statistics [NCES], 2007) and a binary-multilevel logistic regression model analysis. The research questions addressed in this study focus on Asian NNESs:

1. To what extent do parental involvement factors predict the likelihood of Asian NNES student enrollment in a postsecondary institution after controlling for socioeconomic and linguistic factors?

2. If parental involvement factors significantly affect postsecondary attendance after controlling for socioeconomic and linguistic factors, which parental involvement variables are most influential?

This study contributes to the existing literature critically examining relationships between the so-called Asian parenting methods and Asian PSE achievement. Despite the influential factors of socioeconomic and linguistic impacts presented in previous literature, this study explores what other factors such as sociocultural involvement, home language use, as well as cultural values and norms, contribute to Asian NNESs' success or failure; how Asian parents should position themselves in relation to mainstream classrooms and the society; and how policymakers and educators work collaboratively with parents to best support Asian NNESs' academic journeys.

\section{Literature Review}

\section{Relationship Between Parental Involvement and Postsecondary Enrollment}

Research indicates that parental involvement has a significant impact on effect sizes of children's academic achievement, as well as their social and emotional development (Kiang et al., 2013; Li, 2013; Shin, 2009). Greater parental involvement positively correlates with students' academic achievement, attitudes toward learning, self-esteem, peer relations, and PSE enrollment. It negatively correlates with dropout rates (Barton \& Coley, 2007; NCES, 2015a).

Research studies suggest that parental involvement is related to a greater likelihood of attending and graduating from postsecondary institutions (Perna, 2000; Perna \& Titus, 2005). In these studies, however, parental involvement is often operationalized by using merely a single indicator, (i.e., the frequency of discussions between the parents and student about school events) (Perna, 2000). Moreover, research also indicates that the postsecondary enrollment 
process varies across NNESs' ethnic groups (Li \& Wang, 2012; Li \& Yang, 2015; Teranishi, 2010). Researchers have not thoroughly investigated to what extent the relationship between parental involvement and PSE enrollment varies among different population groups (Lim, 2010; Li \& Yang, 2015).

From a sociocultural perspective, differences in PSE enrollment among NNESs can be explained in terms of factors related to their social networks (Dika \& Singh, 2002) and their cultural values (Kiang et al., 2013; Li, 2013). This current study draws on Vygotsky's (1987) sociocultural theory pinpointing the concepts of understanding the relationships and communication among NNESs, parents, educators, school administrators, and home communities. This theoretical framework emphasizes the term "culturally responsive inclusion" recognizing the familial values that shape NNESs' learning experiences and seeking to collaborate with home communities so that both NNESs and their parents feel a sense of belonging and are able to contribute more authentically to their children's formal academic learning (Berryman, Nevin, SooHoo, \& Ford, 2015).

\section{Parental Involvement of Non-dominant Sociocultural Groups}

Despite a large number of studies suggesting that parental participation is an essential factor in academic performance (Li, Hinojosa, Wexler, Bian, \& Matinez, 2017; Zhao, 2011), findings of parental involvement are not consistent across different ethnicities (Lim, 2010). Fan and Chen (2001) conducted a meta-analysis investigating the impact of parental involvement and found that it positively affects academic performance. Results showed that parental expectations for students' academic achievement had the strongest correlation, while parental home supervision had the lowest. Therefore, it is essential to investigate to what extent specific types of parental involvement influence NNESs' academic success.

Different cultural beliefs about education and parenting roles lead to varying attitudes toward attending school events and interacting with teachers and administrators at school (Fuligni \& Fuligni, 2007; Kiang et al., 2013; Li, 2013; Sy, 2006). For instance, Chavkin and Gonzalez (1995) report that many Hispanic immigrant parents believe that they, as parents, should not influence the school's curriculum and instruction. Similarly, Asian immigrant parents often willingly accept teachers and school administrators as authority out of respect, instead of collaborating as equal partners (Li, 2013; Shin, 2009; Sy, 2006). Ethnic minority 
immigrant parents with low SES tend to feel particularly unwelcome in an academic setting as they experience isolation and discrimination (Li, 2013; Li \& Yang, 2015; Shin, 2009).

While the number of the NNESs is increasing, their postsecondary enrollment rate has remained low in comparison to that of NESs. Several studies have addressed NNESs' postsecondary enrollment (Lucas \& Villegas, 2013; NCES, 2015b) and report that compared with NESs, NNESs have higher dropout rates (Menken, 2010; Sheng, Sheng, \& Anderson, 2011), lower graduation rates (Menken, 2010), and overrepresentation in special education programs (Li et al., 2017; Sullivan, 2011). Klein, Burgarin, Beltranena, and McAuthur (2004) claim that in comparison with 18 to 24-year-old NESs, NNESs were less likely than others to enroll in colleges. About the NNESs' educational goals (Kanno \& Cromley, 2011), when compared to NESs, those high school NNESs who have limited English language proficiency are less likely to complete college degrees within eight years of their high school graduation. The achievement gap in postsecondary enrollment between NNESs and NESs is due in part to content areas, notably reading and language arts, which require higher English proficiency (Abedi, 2002; Abedi, Leon, \& Mirocha, 2003; Woo, 2009). While language proficiency has a strong impact on students' content knowledge and academic performance, sociocultural backgrounds, such as parental support (Li, 2013; Li \& Wang, 2012; Uy, 2015), parent-school relationships (Jung \& Zhang, 2016; Uy, 2015), home language literacy (Li, 2013; Li \& Yang, 2015), as well as cultural values and norms (Kiang et al., 2013) are also important and often overlooked.

NNESs are currently ill-served by disciplinary divisions in academic settings that either fail to recognize NNES status as significant or investigate NNESs' high school experiences in terms of sociocultural aspects. There is a dearth of research focused on parental involvement. Parental involvement may be one of the most essential factors for NNESs from families with diverse language and cultural backgrounds. Policy makers and school administrators cannot fully address the needs of NNESs without understanding the impact of parental involvement on PSE enrollment (Kanno \& Harklau, 2012). There is a need to identify to what extent parental involvement influences PSE enrollment from both NNESs' and parental perspectives. This will increase the awareness among policymakers, school administrators, teachers, and parents as to how they can support NNESs throughout their preparation for PSE. 


\section{Impact of Asian Parental Involvement on NNESs' Postsecondary Enrollment}

Asian immigrants have become the fastest-growing racial population, surpassing Hispanic immigrants, in the US (Pew Research Center, 2012). More than 18 million people (6\% of the total US population) are Asian immigrants, and among this population, $74 \%$ of the adults were not born in the US (Pew Research Center, 2012). In the American educational setting, more than $5 \%$ of the total students are Asian immigrants (Gebeloff, Evans, \& Scheinkman, 2012), and this population has been regarded as the best-educated or commonly called the “model minority” (Li \& Yang, 2015; Pew Research Center, 2012; Paik et al., 2017). According to the National Postsecondary Student Aid Study (NPSAS) (Musu-Gillette et al, 2016), among all the ethnic groups, Asian students had the highest postsecondary enrollment rate of $62 \%$ and the highest postsecondary graduation rate of $71 \%$ in 2013 . That number of the postsecondary enrollment was higher than White (42\%), Black (34\%), Hispanic (34\%), Pacific Islander (33\%), and American Indian/Alaska Native (32\%) students, as well as students of two or more races (45\%). However, Asian students in this statistic report included both Asian Americans whose first language is English, and students who are the first or "1.5 generation" (students who were brought to the US at a very young age) and whose first language is not English. These two types of demographics present very different home cultures and values from most other American families. Moreover, few studies take into consideration that Asian populations are comprised of diverse cultural values and norms, as well as language and sociocultural backgrounds.

Census data reveal that a large number of Asian immigrant population succeed in the US academic setting; however, this neglects the diversity of Asian immigrant subgroups and is an overgeneralized concept that falls into the model minority stereotype (Li \& Yang, 2015; Paik et al., 2017). This misleading notion that Asian immigrants are monolithic and have high academic achievements results in neglecting the disparity and gaps existing among these subgroups (Paik, Kula, Saito, Rahman, \& Witenstein, 2014). Asian students are not part of the national literacy crisis because of their high scores in math and literacy as a group. However, a growing number of studies indicate large gaps in academic attainment among a myriad of ethnic groups of the Asian student population (Li \& Yang, 2015). For instance, almost three-quarters of Indians over 25 have a college diploma, while $11 \%$ of Cambodians, Hmong, and Laotians earned a college degree (US Census Bureau, 2010). 
Besides high test scores, there are more important skills, such as collaborative and problem-solving skills, that students need to develop, as a group. These skills should be learned not only at school but also at home. The Programme for International Students Assessment [PISA] reports that in 2015 more than one out of four students in China, Hong Kong, Singapore, and Taiwan are top-performing students in mathematics. Seven out of the top ten countries that scored the highest in science are from Asian countries (PISA, 2018). However, the average across the Organization for Economic Cooperation and Development (OECD) countries shows less than $30 \%$ of students are capable of solving straightforward collaborative problems. Fewer than one out of six students from China, Hong Kong, Japan, Korea, and Singapore are low achievers in collaborative problem-solving tasks.

Parenting styles also vary across Asian cultural values and sociocultural norms. When it comes to Asian parenting, the image of "tiger mother" is a common stereotype, often considered as high parental expectations and demanding parenting. Mixed results showed that while the "Asian model minority myth" has continued to circulate in the media, indicating Asian NNESs" high academic achievement, other research investigates case studies of low-achieving Asian students and the socio-emotional struggle of high-achieving Asian students (Li, 2013). On the one hand, much prior literature reveals the positive impact of high parental educational expectation on NNESs' academic performance. For instance, studies emphasize the role that parents play in transmitting educational values and practices to Asian NNESs. Parents' expectations encourage students to become open to experiences, to bring the home value and culture to academic learning, and to build a sense of self from their engagement in the learning enterprise (Hattie \& Clinton, 2008).

Further, research shows NNESs' academic achievement differs based on NNESs' family resources, cultural values, and backgrounds (Fingerman et al., 2014; Lim, 2010). From a sociocultural perspective, home culture including parents' values and norms toward education can help explain Asian NNESs' academic performance (Fingerman et al., 2014). For instance, cultural values, namely a tradition of respect for teachers, may contribute to the positive value some NNESs' families place on education (Lim, 2010). In addition, NNESs perform better and have higher levels of motivation when parents are supportive, are involved in their children's schooling, encourage their children, and expect academic success, which results in NNESs having higher motivation and being willing to continue studying after high school (Fingerman 
et al., 2014; Lim, 2010). On the other hand, parents and educators should also keep in mind which methods are really good for the next generation, given the widely reported psychological stress and intergenerational conflict among Asian NNESs in the US.

Therefore, parental expectations alone are not sufficient to predict Asian NNESs' academic success. Limited studies have investigated the relationships between parents and students, parents and school, and Asian NNESs' home language use. Research results on the impact of immigrant children's home language and heritage language use on academic achievement remains a complex issue. Some studies indicated that English-only environments help students to perform better at school because students are more focused and able to adjust to the mainstream classroom faster, while other research reveals that students learn better using both their heritage language and target language because it encourages students to associate with their positive cultural and ethnic identities (Lee, 2002, 2014; Li \& Wen, 2015), academic achievements (Chung, 2012), as well as psychological development and mental health (Liu, Benner, Lau, \& Kim, 2009).

Different ethnic groups also have different perspectives on ways to use heritage languages. A study showed that there was a general disinterest among Asian American parents, especially middle-class Chinese and Japanese immigrant families, to pass down cultural values (e.g., their heritage language use) to their children in order to prevent racism attacks (Tuan, 1998). In addition, reports reveal that only four out of ten second-generation Asian Americans speak their heritage language fluently, compared to eight out of ten second-generation Hispanic Americans. For the third generation of Asian Americans, only 10\% preserved their heritage language, while $40 \%$ of Hispanic Americans were able to preserve their parents' heritage language (Li \& Wen, 2015; Portes \& Rumbaut, 2006).

The present study fills the gap in the literature by examining the multiple parental involvement factors of the Asian NNESs across various Asian groups and by taking into consideration factors such as sociocultural involvement, home culture, and home language use. The study further discusses and challenges the previous literature and theories regarding the impact of parents' educational expectations and home language literacy on Asian NNESs' PSE enrollment. 


\section{Theoretical Framework}

This study is framed and supported by two major theories: (1) the Vygotskian sociocultural theory (SCT) (Lantolf \& Thorne, 2006; Vygotsky, 1978, 1987); and (2) the six concepts of parental involvement by Epstein (Epstein \& Sanders, 2002).

\section{The Vygotskian Sociocultural Theory}

Vygotsky's sociocultural theory (Vygotsky, 1978, 1987) describes human learning as a social process and credits the development of human intelligences to society and culture. To Vygotsky, everything is learned through social interactions with others first, and then through individual internalizations.

Every function in the child's cultural development appears twice: first, on the social level, and later, on the individual level; first, between people (interpsychological) and then inside the child (intrapsychological). This applies equally to voluntary attention, to logical memory, and to the formation of concepts. All the higher functions originate as actual relationships between individuals. (Vygotsky, 1978, p.57)

Vygotsky's theory frames the theme of this study: the exploration of the relationships among Asian NNESs, their parents, schools, and communities, and supports the investigation of how society and home impact the PSE of Asian NNESs. Despite the importance of sociocultural theory, in order to set criteria for selecting parental involvement variables, this study follows Epstein's six types of parental involvement elements.

\section{Epstein's Concepts of Parent Involvement}

Epstein and Sanders (2002) present a research-based framework: the six types of school, family, and community interactions, to assist schools in developing school and family partnership programs for student success.

Epstein's Framework (2002) encompasses: (1) parenting: establish home environments to support children as students; (2) communicating: effective forms of school-to-home and home-to-school communications; (3) volunteering: recruit and organize parent help and support; (4) learning at home: help students at home with homework and other curriculum-related activities, decisions, and planning; (5) decision-making: participants in school decisions; and (6) 
collaborate with community: use community resources for families, students, and the school, and provide services to the community.

Parental involvement factors were selected for this study based on Epstein and Sanders' (2002) six concepts of parental involvement and were measured by parental participation in the student's life in both home and school settings. The following four types of parental involvement factors are examined: (1) Parent-School Involvement, (2) Parent-Student Involvement, (3) Home Culture, and (4) Home Language Environment (see detailed description in Table 1).

\section{Research Methods and Data Sources}

The section begins by describing the Education Longitudinal Study (ELS) dataset, the sample selection procedures, and the variables selected. ELS is used in this study because it is a longitudinal study that captures the trajectory of students' growth in their academic careers; it includes a myriad of familial factors and background information of students' families, school environments, and local communities; and it contains diverse ethnic groups of students. The appropriateness of the selection variables for the present inquiry is also discussed. The binary logistic regression model is applied in this study. The proposed analytic techniques and statistical model are used to provide a rationale for the variable selection and analysis methodology. More specifically, access to these variables may help explain what factors affect whether Asian NNESs enroll in PSE.

\section{Data}

This study, being a follow-up on a previous work (Yeh, 2014) that explored parental involvement and NNESs' PSE in the US, uses the nationally representative data from the Education Longitudinal Study dataset in 2002 [ELS: 2002] (Ingels et al., 2007; NCES, 2007). ELS: 2002 conducted surveys in the same units (schools and students) repeatedly over time. The students surveyed were followed at four time periods, including a base-year interview with 10th-grade students in 2002; a two-year interval follow-up with high school seniors in 2004; a second follow-up with college sophomores in 2006; and the last follow-up data were collected eight years after high school, in 2012. ELS: 2002 is a multilevel study that involved multiple participant populations, such as students, parents, teachers, and schools. The dependent variable indicates the level of the respondent's first-attended postsecondary institution between 2004 and 
2006. The current study focuses on Asian NNESs who began PSE within two years of graduation in 2002. Therefore, this study includes data from the base year and second follow-up in 2006. The base-year interview was carried out in a nationally representative probability sample of 752 public, Catholic, and other private schools during the 2001-2002 school year. From a total of the 17,591 eligible students, selected students (about 15,362) completed a baseyear questionnaire, for a weighted response rate of $87 \%$ (Ingels et al., 2007).

\section{Sample}

This study is an expansion of author's dissertation study. The sample in this study consisted of 991 sophomore-cohort Asian NNESs, collected from the base-year survey through the second follow-up. This current study adopts the similar methodology from previous research investigating overall NNESs population and different ethnic groups (Yeh, 2014). The total population of NNESs was 2,586 in 523 schools, and NNESs represented 13.5\% (weighted) of the sophomore-cohort, base-year respondents (Ingels et al., 2007). Asian NNESs were defined by the National Center for Educational Statistics (2007) as one of five distinctive categories of NNESs' ethnic origin. ${ }^{i}$ Asian students include Native Hawaiian or other Pacific Islander. The total sample size for the main analyses of Asian NNESs before applying weights was 991. This represents $38.30 \%$ (unweighted) and $20.50 \%$ (weighted) of sophomore-cohort, base-year NNES respondents. For each analysis, the weighted sample size will be reported. The sample weight was limited to Asian NNESs who responded in the second follow-up in 2006, weighted from spring $200210^{\text {th }}$-grade students to 2006 (labelled F2BYWT ELS:2002 database) (Yeh, 2014). The researcher defined Asian NNESs based on students' responses to the question, "Whether English is student's first language." The students who reported that English is not their native language were coded as Asian NNESs.

\section{Dependent Variable}

The dependent variable was postsecondary school enrollment (Yes = enrolled in PSE / No $=$ did not enroll in PSE) and was measured at the student level (see Table 1). The dependent variable in the current study was identified as:

the level of offering of the first postsecondary institution (labeled as F2EVRATT in ELS:2002), indicating the level of the respondent's first-attended postsecondary 
Bridging the Gaps

institution between 2004 and 2006. The variable is a two-category measure: (1) did not attend postsecondary education and (2) attended a postsecondary education (i.e., two-year college/university, four-year college/university) (Yeh, 2014, p.78).

\section{Independent Variables}

Variable selection for independent variables was based on Epstein and Sanders' (2002) six concepts of parental involvement: parenting, communication, volunteering, learning at home, collaboration with the community, and decision making. The following section introduces the independent variables: parental involvement factors as the main variables, and two sets of covariates in terms of linguistic and socioeconomic factors (see Table 1 and Appendix A). 
Table 1: Means and Standard Deviations of the Variables: Asian Non-native English Speakers (Adopted Table from Yeh, 2014)

Variable name Asian NNESs M SD

$(\mathrm{N}=991)$

Parental involvement (Student level)

Parent-School Involvement: 1

1.23

Parent contacted the school about NNESs' academic performance

Parent contacted school about plans after high school

Parent contacted the school about NNESs' course selection

Parent-School Involvement 2:

1.10

.37

Students have problematic behavior at school

Parent-School Involvement 3:

$1.21 \quad .50$

Parent reports contacting the school doing volunteer work

\begin{tabular}{lll}
\hline Parent-Student Involvement & 2.08 & .48
\end{tabular}

Parent-student discussions about education-related issues, and parent's advice for NNESs' school work

Home Culture 1:

$5.50 \quad 1.35$

Parent's Educational Expectations

Home Culture 2 GPA:

Parent's values/norms toward education: has rules about GPA

Home Language Environment 1:

3.29

.89

NNESs' Frequency of Native Language Use

Home Language Environment 2:

Parent speaks language other than English at home

Covariates: SES Factors (School level)

Mean Income

8.75

1.25

Mean Teacher Quality

39.26

20.81

Covariates: SES Factors (Student level)

Family Income

$8.00 \quad 2.75$

Family Resources

Parents' Level of Education 
Linguistic Factors (Student level)

Reading Test Standardized Score (IRT)

50.08

10.11

NNESs' self-reported English language ability

1.34

.55

1.44

.62

1.46

.62

1.53

.68

Note: Results are weighted (weighted variable $=$ F2BYWT) to yield population estimates.

Parental Involvement Factors. A large number of research studies assert the important role of parental involvement in NNESs' academic success from kindergarten through twelfth grade (Chang, Park, Singh, \& Sung, 2009; Jeynes, 2012; Meza, 2011) rather than focus on impacts of PSE enrollment. Therefore this study, exploring the degree to which NNESs' parental involvement affects academic success at the postsecondary level, contributes to the research literature on parental involvement variables in PSE enrollment. Parental involvement factors were selected based on Epstein and Sanders' (2002) six concepts of parental involvement and were measured by parental participation in the student's life in both home and school settings. The following four types of parental involvement factors are examined: (1) Parent-School Involvement, (2) Parent-Student Involvement, (3) Home Culture, and (4) Home Language Environment (see detailed description in Table 1).

Socioeconomic Factors. PSE affordability is the main reason low-income and minority students do not enroll in PSE (Heller, 2001, 2002; Perna, 2002). Long and Riley (2007) investigated how low-income students and minority students face significant financial obstacles in attending PSE. Their study suggested that it is important to provide more need-based financial aid for these groups of students, particularly scholarships and grants to decrease the cost of PSE as a barrier to PSE enrollment. The covariates of SES factors included mean income, mean teacher quality, family income, family resources, and parents' level of education (see Table 1).

Linguistic Factors. Linguistic factors are influential factors impacting NNESs' PSE enrollment; however, with parental involvement and familial support, the likelihood of NNESs' academic success could be greater. Therefore, the current study controlled for the linguistic factors, such as grade reading scores and self-reported English language ability, as covariates (see Table 1). 


\section{Analytic Techniques}

The ELS: 2002 dataset was collected with a sampling design that involved more than one level (i.e., sampling students from sampled schools); therefore, multilevel logistic regression modeling is appropriate and applicable (Ingels et al., 2007).

To answer the first and second research questions about the extent to which parental involvement contributes to the likelihood of Asian NNESs' PSE enrollment and which variables are most influential, the current study built four models to analyze the data. The first was an unconditional model that encompassed only the outcome measure without any independent variables at either the student or school levels. The purpose of this model was to examine whether significant variance occurs between schools and provide a standard for comparing with the later models. The second model included a series of SES covariates. This model examined which SES variables had the strongest impact in Asian NNESs' PSE enrollment. The third model included a series of linguistic covariates. This model examined which linguistic variables had the strongest impact in NNESs' PSE enrollment (Yeh, 2014). The last model included a series of parental involvement predictors. In the current study, these different models were analyzed for Asian NNES populations.

\section{Statistical Model}

The present study used logistic regression to estimate the likelihood that what type of parental involvement predicts Asian NNES's PSE enrollment: the dichotomous variable of Asian NNESs' reporting Yes (enrolled in PSE) or No (did not enroll in PSE). Logistic regression resolves the violated multiple linear regression assumptions by attempting to predict not the dichotomous variable, but the probability of belonging to the treatment case (those who report enrolling in PSE). Equation 1 is the formula for the logistic regression:

$\hat{Y}_{i}=\frac{e^{u}}{1+e^{u}}$

Where $\hat{Y}_{i}$ is the estimated probability that the $i$ th case $(i=1, \ldots, n)$ denotes the student.

$u$ is a linear regression equation of the form indicated in Equation 2:

$\mathrm{u}=\mathrm{A}+\mathrm{B}_{1} \mathrm{X}_{1}+\mathrm{B}_{2} \mathrm{X}_{2}+\ldots+\mathrm{B}_{\mathrm{k}} \mathrm{X}_{\mathrm{k}}$

Where $\mathrm{A}$ is the constant, $\mathrm{B}_{\mathrm{k}}$ is the coefficient for the $k$ th predictor and $X_{k}$ is the predictor (Tabachnick \& Fidell, 2006).

The following section demonstrates four models described above.

(1) An unconditional model: 
$\mathrm{u}=\mathrm{A}$

(2) Model for SES factors:

$u=A+\beta_{1}\left(\right.$ Asian BYSTLANG) $+\beta_{2}$ (Asian ECONO)

(3) Model for linguistic factors:

$u=A+\beta_{1}($ Asian BYSTLANG $)+\beta_{2}($ Asian ECONO $)+\beta_{3}$ (Asian LING)

(4) Model for parental involvement factors:

$u=A+\beta_{1}($ Asian BYSTLANG $)+\beta_{2}\left(\right.$ Asian ECONO) $+\beta_{3}$ (Asian LING) $+\beta_{4}$ (Asian PARENT) Where

$\beta_{1}=$ Native language status slope; Native language status is defined as Asian

BYSTLANG $=$ Whether English is Asian student's native language-composite.

Asian ECONO = is a category of variables: (1) mean income; (2) mean teacher quality;

(3) family income; (4) family resources; and (5) parents' level of education.

Asian LING =is a category of NNESs' English language proficiency in terms of (1) 10th grade reading scores (IRT); and (2) NNESs' self-reported English language ability.

Asian PARENT = is a category of (1) parent-school involvement; (2) parent-student involvement; (3) home culture; and (4) home literacy environment.

\section{Results}

The descriptive statistics of all measurement variables are provided in Table 1 for the total sample. Table 1 shows four categories of independent variables in terms of parent-school involvement, parent-student involvement, home culture, and home language environment.

\section{Binary Logistic Regression Model for Asian NNES Populations}

A binary logistic regression analysis was performed to explore to what extent parental involvement factors predict the likelihood of Asian NNESs' PSE enrollment after controlling for SES and linguistic factors among Asian ethnic groups. The outcome variable, whether enrolled in PSE, was coded $0=$ No and $1=$ Yes. Data from 836 cases were included in this analysis (155 cases were missing for the outcome variable from the total of 991 cases after applying the weights).

To answer the research question about to what extent parental involvement factors predict the likelihood of Asian students enrolling in a postsecondary institution after controlling for SES and linguistic factors, a binary logistic regression analysis was performed with an unconditional model (the null or constant-only model), which encompassed merely the outcome 
measure without any independent variables at either the student or school levels, and three blocks (1) ten SES variables were entered in the first block; (2) five linguistic variables were entered in the second block; and (3) eight parental involvement variables were entered in the third block (Yeh, 2014).

The Block 0 results are the null or constant-only model. The null hypothesis for the constant of the null model is that the odds of enrolling in PSE versus not enrolling in PSE for the entire sample is one ( $N=836$ NNESs; 7 cases were missing from the total 843 cases of the outcome variable after applying the weights). The value of $B_{0}$ differs significantly from 0 ( $B_{0}=$ 1.58 , Wald $\left.\chi^{2}(1, N=836)=28117.76, p<.001\right)$. This tells us that the odds of enrolling in PSE for the overall sample differed significantly from 1.00. Model 1 correctly classifies cases $61.80 \%$ of the time.

The Block 1 results appear in Table 2 and refer to the second model with the series of SES covariates. The second model's comparison with a null model was statistically significant, $\chi^{2}(10)=3131.90, p<.001$. This significance indicates that together SES variables were significantly related to enrollment in PSE. Model 2 includes ten additional theoretically important independent SES variables. According to the likelihood ratio test statistic, Model 2 is superior to the null model (Model 1) in terms of overall model fit, Nagelkerke's $R^{2}=.14$. Model 2 correctly classifies cases $65.20 \%$ of the time. Table 2 presents the raw score binary logistic regression coefficients (B), standard error of the estimate (SE), Wald statistics, and the estimated change in odds of enrolling in PSE [i.e., the odds ratio labeled Exp (B)], along with a 95\% CI. For each coefficient, a Wald chi-square was calculated. All coefficients were statistically significant, except "Family Resources_family has regularly received magazine" (see Table 2, item BYS84B). 
Bridging the Gaps

Table 2: Binary Logistic Regression Model for Predicting Asian Students' PSE Enrollment ( $N=$ 836)



Nagelkerke

Block 1

7131.897

.142

Family Resources

BYS84A

BYS84B

$-.155 \quad .022$

50.805

$.000 \quad .856$

BYS84C

$.004 \quad .022$

.039

$.844 \quad 1.004$

BYS84D

$.901 \quad .041$

481.005

.000

2.463

BYS84E

$.636 \quad .035$

322.298

$.000 \quad 1.888$

BYS84H

$-.059 \quad .025$

5.646

.017

.943

Family Income

BYP85

.019

$.004 \quad 20.687$

.425

.999

Parents' Level of Education

BYP34A

.131

$.005 \quad 609.574$

.000

1.141

Mean Teacher Quality

F1A37D

$\begin{array}{lll}-.002 & .000 & 10.280\end{array}$

.001

.998

Mean Income

$\begin{array}{lllllll}\text { ALL SCHOOLSES } & .181 & .009 & 363.511 & .000 & 1.198\end{array}$

Block 2

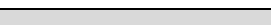

Reading Test Standardized Score

BYTXRSTD

$$
.057
$$

$.001 \quad 1795.433$

.000

1.059

Self-Reported English Ability

\begin{tabular}{lrrrrrrl} 
BYS70A & -.500 & .031 & 258.809 & .000 & .607 & & \\
BYS70B & .570 & .035 & 270.530 & .000 & 1.769 & & \\
BYS70C & .060 & .033 & 3.204 & .073 & 1.062 & & \\
BYS70D & -.174 & .028 & 38.220 & .000 & .840 & & \\
\hline Block 3 & & & & & & 1445.855 & .027 \\
Parent Students & .671 & .024 & 780.837 & .000 & 1.955 & & \\
Parent School 2 & -.378 & .029 & 170.961 & .000 & .685 & \\
Parent School 3 & .298 & .029 & 103.934 & .000 & 1.348 & \\
Home Culture 2 & .247 & .032 & 59.197 & .000 & 1.280 &
\end{tabular}




\begin{tabular}{lrrrrr} 
Home Language 2 & .117 & .017 & 47.457 & .000 & 1.124 \\
Parent School 1 & -.135 & .030 & 20.875 & .000 & .874 \\
Home Culture 1 & .047 & .008 & 35.380 & .000 & 1.048 \\
Home Language 1 & .019 & .014 & 1.840 & .175 & 1.019 \\
\hline Constant & -4.904 & .138 & 1269.862 & .000 & .007 \\
\hline
\end{tabular}

Note. Estimated regression coefficients at entry.i Degrees of freedom for all Wald statistics is 1. $\operatorname{Exp}(B)=$ odds ratio; $C I=$ confidence interval; $S E=$ standard error; Sig. = significance level .

The Block 2 results in Table 2 are results for a third model with a series of linguistic covariates included in the model from the previous block. The third model's comparison to the second model was statistically significant, $\chi^{2}(5)=2580.88, p<.001$. This indicates that together linguistic variables were related to PSE in the presence of SES variables. Model 3 includes five additional theoretically important independent variables: linguistic variables. According to the likelihood ratio test statistic, Model 3 is superior to Model 2 in terms of overall model fit, Nagelkerke's $R^{2}=.19$. Model 3 correctly classifies cases $68.20 \%$ of the time. All coefficients were statistically significant, except "How well 10th grader reads English," "Family Resources, family has DVD player," and "Family Income" (Table 2, items BYS70C, BYS84D, and BYP85).

The Block 3 results in Table 2 are results for a fourth model with a series of parental involvement variables included in the model from Block 2. This final model introduced parental involvement measures and examined the effects of parental involvement measures after controlling for SES and linguistic variables. The fourth model's comparison to the third model was statistically significant, $\chi^{2}(8)=1480.24, p<.001$. This indicates that together parental involvement variables were related to PSE in the presence of SES and linguistic variables. Model 4 includes eight additional theoretically important independent variables: parental involvement variables. According to the likelihood ratio test statistic, Model 4 is superior to Model 3 in terms of overall model fit, Nagelkerke's $R^{2}=.22$. Model 4 correctly classifies cases $70.50 \%$ of the time. All coefficients were statistically significant, except "NNESs' frequency of native language use" and "How well 10th-grader speaks English" (See Table 2, items BYS69A and BYS70B). This provides some evidence to address the second research question (See Appendix A for the output of Binary Logistic Regression to predict enrolling in Asian students' PSE. Model 4 added parental involvement variables). 
A review of the statistically significant odd-ratios from Table 2 shows that the odds of enrolling in PSE relative to not enrolling increased with frequency of Parent-Student Involvement $(O R=1.96 ; 95 \% \mathrm{CI}=[1.87,2.05])$, and shows that the odds of enrolling in PSE were about 1.96 times higher for those whose parents were involved in students' academic lives than for those whose parents were not involved in students' academic lives. Parent-School Involvement 2 , Bad Behavior $(O R=.69 ; 95 \% \mathrm{CI}=[.69, .73])$, shows that the odds of enrolling in PSE were about 1.45 times greater per unit of the behavioral problem scale. Parent-School Involvement 3, Parent Volunteer $(O R=1.35 ; 95 \% \mathrm{CI}=[1.27,1.43])$, shows that the odds of enrolling in PSE were about 1.35 times higher for the NNESs whose parents contacted the student's school more often about doing volunteer work than those whose parents contacted the school less. Home Culture 2, GPA $(O R=1.280 ; 95 \% \mathrm{CI}=[1.20,1.36])$, shows that the odds of enrolling in PSE were about 1.28 times higher for those NNESs whose parents have rules about GPA at home than those whose parents did not. Parent-School Involvement $1(O R=.87 ; 95 \%$ $\mathrm{CI}=[.83, .93])$ shows that the odds of enrolling in PSE were about 1.15 times higher for those whose parents contacted the school less often for academic performance, plans after high school, and course selection than those whose parents contacted school more often.

Home Language Environment $2(\mathrm{OR}=1.12 ; 95 \% \mathrm{CI}=[1.09,1.16])$ shows that the odds of enrolling in PSE were about 1.12 times higher for the NNESs whose parents spoke more native language with their children than for those whose parents spoke less native language with their children. In contrast, measures of Home Language Environment 1 were not significant predictors of enrolling in PSE in the presence of the other predictors. Home Culture $1(O R=$ $1.05 ; 95 \% \mathrm{CI}=[1.03,1.06])$ shows that the odds of enrolling in PSE were about 1.05 times higher for Asian NNESs whose parents have one unit higher educational expectations than those whose parents have less educational expectations.

\section{Discussion}

The findings imply that parental involvement practices not only take place at school, but also in the home and community (Epstein \& Sanders, 2002; Sy, 2006); therefore, parents should support their children's academic success by collaborating with the school and community. For Asian NNESs, these results reveal that parental involvement factors have significant influence and implications for PSE enrollment. The findings show that parental involvement factors are related to the likelihood of PSE enrollment, even after controlling for SES and linguistic factors. 
These results are in line with previous work done by Lee and Manning (2001), Shin (2009), and Sy (2006), who found that parenting roles lead to varying attitudes towards parental involvement and are related to a greater likelihood of attending PSE institutions.

Among the eight parental involvement factors, seven variables were statistically significant predictors of Asian NNESs' PSE enrollment after controlling for SES and linguistic factors. Only one variable was not statistically significant: Home Language Environment 1 (NNESs' frequency of native language use). The following discussion addresses the impactful predictors and one seemingly contradictory factor on PSE enrollment (i.e., Parent-School Involvement 1). Parent-Student Involvement, Parent-School Involvement 2, and Parent-School Involvement 3 are the top three strongest factors for PSE enrollment, followed by Home Culture categories and Home Language Environment categories.

According to the results, Parent-Student Involvement (i.e., parent-student discussions about education-related issues) and Parent-School Involvement 2 (i.e., students have problematic behavior at school) have the largest impact on PSE enrollment. Parent-Student Involvement refers to discussion among parents and Asian NNESs on education-related issues and parents' advice for NNESs' on school work. The students' PSE enrollment is higher if their parents advise on their school work, such as discussing school courses, grades, preparation for ACT/SAT, and other issues about PSE. Regarding Parent-School Involvement 2, the result shows that Asian NNESs' enrollment is higher if they perform better and have less bad behavior at school. This finding suggested that Asian NNESs' parents should pay more attention to children's behavior at school and contact the school more often regarding behavioral problems at school.

Parent School Involvement 3 (i.e., parent reports contacting the student's school about doing volunteer work) had the third strongest impact on PSE enrollment. The results show that Asian NNESs' enrollment is higher if Asian NNESs' parents participate in school volunteer work and engage in school activities and events, so that parents understand their students' school activities and environments better.

Home Culture categories and Home Language Environment categories were not the strongest parental involvement factors, but the results still showed that they are significant factors impacting Asian NNESs' PSE enrollment. Home Culture 1 (i.e., parent's educational expectations) refers to how well parents expect Asian NNESs to do in school. Asian NNESs' 
PSE enrollment is higher if their parents have higher educational expectations. In addition, Asian NNESs are more likely to enroll in PSE if their parents' values and norms toward education are higher, such as having strict rules or expectations for 10th graders about maintaining a certain grade point average or performance at school.

Regarding Home Language Environment (i.e., NNESs' frequency of native language use), the study shows that Asian NNESs' enrollment is higher if Asian NNESs speak their native language with their parents. These findings correlate with previous literature that first language use can positively influence NNESs' learning of their second language and help to develop their cognitive and academic language proficiency (Cummins, 1879; 1981).

The findings indicate that Parent-School Involvement 1 (i.e., parents contacted the school about NNESs' academic performance, plans after high school, and course selection) has a negative relationship on PSE enrollment. This may be due to Asian parents contacting the school more frequently when their children have behavior problems or do not perform well at school, rather than having a direct impact on PSE enrollment.

\section{Implications}

This study suggests that the PSE preparation programs designed to promote increased PSE enrollment should recognize that the relationship between parental involvement and PSE enrollment varies across ethnic groups. Mehan, Hubbard, Lintz, and Villanueva (1996) claim that parents with low SES who had high academic expectations usually had insufficient knowledge and resources to help their NNES students with PSE goals. Lopez, Scribner, and Mahitivanichcha (2001) suggest that PSE preparation programs should focus on diversity across ethnic groups, rather than forcing a one-size-fits-all approach. Researchers and educators should take into consideration the diverse social and cultural contexts of each ethnic group when creating PSE programs that assist NNESs. Different ethnic groups need different strategies and support in PSE preparation programs, and additional support is needed for disadvantaged minority families. In order to incorporate parental involvement in the curriculum, school administrators, policy makers, teachers, and parents need to recognize the differences of parents in their expectations, values, and attitudes toward education.

For the Asian population specifically, the findings show several implications for parents and school policy. The Parent-Student Involvement factor is the strongest variable affecting Asian NNESs' PSE enrollment. This suggests that parents should communicate frequently with 
their children, as well as invest time in them so that they can build a healthy relationship with their children. They should assist NNESs with school work and discuss future academic plans. Further, given the findings for Parent-School Involvement variables, it is essential for school administrators and teachers to understand the importance of collaborating with and engaging parents in academic events and in volunteering at school. Previous research suggested Asian NNES parents are more reluctant to communicate with teachers, volunteer at school events, and make decisions for school events. As a result, many Asian NNES parents' concerns were not addressed by school and they preferred taking action outside of school (Li, 2013). For instance, Asian NNES parents send their children to private tutors or "cram schools" to pursue their own educational belief instead of reaching out to the school.

In Li's (2006) study, some parents took outside school more seriously than regular classes. That study also suggests that Asian parents around the world have limited involvement in school and are more passive in building relationships with teachers and schools (Li, 2006). The study suggests that schools should provide opportunities and services to accommodate and support NNES parents' needs so that parents are more willing to attend teacher-parent conferences, volunteer for school events, initiate conversation, and ask for assistance.

The first step for administrators and teachers is to create friendly environments for parents without pressure of being in the spotlight or being forced to mingle or network with strangers. For some Asian NNES parents, they avoid attending school events because they feel anxious about talking to strangers. A space for parents who share similar backgrounds and cultures could reduce anxiety. Having translators if needed, but also just having a point person or familiar faces are good approaches for parents to be more inclined to attend school events. How to access to information and who to reach out to when their children encounter problems are frequently asked questions. Informal workshops with smaller sized groups should be hosted for NNES parents. Allowing more time for questions and answers or even one-on-one time for questions and answers are crucial for NNES parents. For some parents who are not comfortable speaking in public in their second language, this opportunity offers more time to think about what they want to ask and ask more specific questions about their own kids. Through these workshops, schools should encourage parents to be aware of NNESs' behavior at home and school by interacting with school and should not hesitate to contact school while students need 
additional assistance. Future studies need to explore these sociocultural factors that hinder Asian NNES parents' actively engage in school activities and building relationships.

For Home Culture variables, parents' educational expectations and values toward education have a strong impact on PSE enrollment. Parents may establish clear academic expectations for their students, such as maintaining a certain GPA. However, given the widely reported psychological stress and intergenerational conflicts between children and parents (Li, 2013), Asian NNES parents need to keep in mind that for many high-achieving Asian NNESs this could be the cause of too much pressure from family values and expectations. School administrators and teachers should be aware of these hidden norms and cultural values so that they can better understand parents' reactions and students' behaviors. Considering these important cultural factors, schools should provide more opportunities for professional development focusing on culturally responsive teaching (Gay, 2010) and intercultural communication (Byram \& Feng, 2005).

For Home Language Environment variables, NNESs should be encouraged to speak their native language with their parents more often. This result also correlates with previous literature that keeping NNESs' native language use led to less alienation between children and immigrant parents, maintaining communication rifts among family members, preventing cultural loss for NNESs (Li \& Wen, 2015), better academic achievements (Chung, 2012), and less psychological and mental health issues (Liu et al., 2009). Teachers should incorporate culturally responsive teaching methods and meaningful intercultural communicative materials into the curriculum and encourage students to use their own diverse and unique backgrounds to connect to the school content and subjects. Schools should also provide platforms and events for students to explore, discuss, showcase, and celebrate diversity, equity, and inclusion.

Implications for policy makers include (1) providing community-based professional development (Uy, 2015) for administrators and educators on how to apply culturally responsive teaching and strategies while working with Asian NNESs' families; (2) creating a committee to offer feedback and advice for schools to understand how to build meaningful relationships with Asian NNES parents and translate documents to reduce the language and cultural barriers; and (3) developing programs for Asian NNES parents to understand the American educational systems and encouraging them to take on leadership roles in school events. 


\section{Limitations and Future Directions}

Perna (2007) claims that in order to eliminate the persistent gap among ethnic groups in PSE enrollment, policymakers and school administrators need to trace the sources of the gaps. It is also important to gain a comprehensive understanding of the PSE enrollment patterns across various ethnic groups and how students of different races make decisions about whether to enroll in PSE institutions. In this sense, future studies about Asian NNESs' academic $^{1}$ and psychological variables at the student and school levels are needed. This would provide additional information for policymakers, education researchers, teachers, and parents to further understand to what extent parental factors affect the PSE enrollment across ethnic groups. The findings of this study can be used to guide educators in their efforts to narrow the persisting gaps in PSE enrollment among ethnic groups.

NNESs' language use and proficiency are affected by their home language use and cultural background. Thus, future studies should take into consideration both Asian NNES students' and parents' generational status. Generational status (i.e., time spent in the US, age at arrival in the US, and number of years of English learning) may be an important factor in PSE enrollment as it influences parents' motivation and educational expectations for NNESs and should be investigated in future studies. These factors illustrate the complex nature of the relationship between generational status and NNESs' academic achievement. The ELS: 2002 dataset includes information at multiple levels: not only information from both students and schools, but from students' parents, teachers, and school administrators. Therefore, the Hierarchical Linear Model (HLM) (Raudenbush \& Bryk, 2002) could be used to conduct future research with the dataset of the ELS: 2002. Future studies should also investigate, more specifically, the size of the relationships and the differences across ethnic groups comparatively. With continuing research, the results of this and future studies can be used to help provide more opportunities for students from all cultural backgrounds to enroll in postsecondary education.

${ }^{1}$ Student's academic variables, such as transcripts, are in the ELS restricted dataset. 


\section{References}

Abedi, J. (2002). Standardized achievement tests and English language learners: Psychometrics issues. Educational Assessment, 8(3), 231-257.

Abedi, J., Leon, S., \& Mirocha, J. (2003). Impact of students' language background on content-based assessment: Analyses of extant data (CSE Tech. Rep. No. 603). Los Angeles: University of California, National Center for Research on Evaluation, Standards, and Student Testing.

Barton, P. E. \& Coley, R. J. (2007). The family: America's smallest school. Princeton, NJ: Educational Testing Services.

Berryman, M., Nevin, A., SooHoo, S., \& Ford, T. (2015). Culturally responsive contexts: Establishing relationships for inclusion. International Journal of Special Education, 30(3), $1-13$.

Byram, M., \& Feng, A. (2005). Teaching and researching intercultural competence. In E. Hinkel (Ed.), Handbook of research in second language teaching and learning (pp. 911-930). Mahwah, NJ: Lawrence Erlbaum Associates, Inc.

Chang, M. L., Park., B., Singh, K., Sung, B. Y. (2009). Parental involvement, parenting behaviors, and children's cognitive development in low-income and minority families. Journal of Research in Childhood Education, 23(3), 309-325.

Chavkin, N. F., \& Gonzalez, D. L. (1995). Forging partnerships between Mexican American parents and the schools. West Virginia: ERIC Clearinghouse on Rural Education and Small Schools. (ERIC Document Reproduction Service No. ED388489).

Chung, J. (2012). The relationship of heritage language/culture education with academic achievement: A study of the 1.5 and 2nd generation of Korean American high school students. Unpublished doctoral dissertation, University of Oregon.

Cummins, J. (1979). Linguistic interdependence and the educational development of bilingual children. Review of Educational Research, 49(2), 222-251.

Cummins, J. (1981). The role of primary language development in promoting educational success for language minority students. In C. F. Leyba (Ed.), Schooling and language minority students: A theoretical framework (1st ed., pp. 3-47). Los Angeles: Evaluation, Dissemination and Assessment Center, California State University.

Dika, S. L., \& Singh, K. (2002). Application of social capital in educational literature: A critical synthesis. Review of Educational Research 72, 31-60.

Epstein, J. L., \& Sanders, M. G. (2002). Family, school, and community partnerships. In M. Bornstein (Ed.), Handbook of parenting, Volume 5: Practical issues in parenting (2nd ed., pp. 407-438). Mahwah, NJ: Lawrence Erlbaum Associates.

Fan, X., \& Chen, M. (2001). Parental involvement and students' academic achievement: A meta-analysis. Educational Psychology Review, 13, 1-22.

Fingerman, K., Cheng, Y. P., Kim, K., Fung, H., Han, G., Lang, F., Lee, W., \& Wagner J. (2014). Parental involvement with college students in Germany, Hong Kong, Korea, and the United States. Journal of Family Issues, 37(10), 1384-1411.

Fuligni, A. J., \& Fuligni, A. S. (2007). Immigrant families and the educational development of their children. In J. E. Lansford, K. Deater-Deckard, \& M. H. Bornstein (Eds.), Immigrant families in contemporary society (pp. 231-249). New York: Guildford Press.

Gándara, P., \& Rumberger, R. W. (2009). Immigration, language, and education: How does language policy structure opportunity? Teachers College Record, 111(3), 750-782. 
Gay, G. (2010). Culturally responsive teaching: Theory, research, and practice. New York: Teachers College Press.

Gebeloff, R., Evans, T., \& Scheinkman, A. (2012). Diversity in the classroom. The New York Times. Retrieved from http://projects.nytimes.com/immigration/enrollment

Hattie, J. A. C., \& Clinton, J. (2008). Identifying accomplished teachers: A validation study. In L. Ingvarson \& J. A. C. Hattie (Eds.), Assessing teachers for professional certification: The first decade of the National Board for Professional Teaching Standards (pp. 313-344). Oxford, UK: Elsevier.

Heller, D. E. (Ed.). (2001). The states and public higher education policy: Affordability, access, and accountability. Baltimore, MD: Johns Hopkins University Press.

Heller, D. E. (Ed.). (2002). Condition of access: Higher education for lower income students. Westport, CT: American Council on Education/Praeger.

Her, C. S. (2014). Ready or not: The academic college readiness of Southeast Asia Americans. Multicultural Perspectives, 16(1), 35-42.

Ingels, S. J., Pratt, D. J., Wilson, D., Burns, L. J., Currivan, D., Rogers, J. E., et al. (2007). Education Longitudinal Study of, 2002: Base-year to second follow-up data file documentation (NCES, 2008-347). Washington, DC: US Department of Education, National Center for Education Statistics.

Jeynes, W. (2012). A meta-analysis of the efficacy of different types of parental involvement programs for urban students. Urban Education, 47(4), 706-742.

Kanno, Y., \& Cromley, J. (2011). English language learners' college access and attainment: A national level analysis. Paper presented at the American Association for Applied Linguistics. Chicago, IL.

Kanno, Y., \& Harklau, L. (2012). Linguistic minority students go to college: Preparation, access, and persistence. New York: Routledge.

Klein, S., Burgarin, R., Beltranena, R., \& McAuthur, E. (2004). Language minorities and their educational and labor market indicators: Recent trends. NCES, 2004-009. Retrieved from National Center for Educational Statistics: http://nces.ed.gov/pubsearch/pubsinfo.asp?pubid=2004009

Kiang, L., Andrews, K., Stein, G., Supple, A., \& Gonzalez, L. (2013). Socioeconomic stress and academic adjustment among Asian American adolescents: The protective role of family obligation. Journal of Youth \& Adolescence, 42(6), 837-847.

Lantolf, J. P., \& Thorne, S. L. (2006). Sociocultural theory and the genesis of second language development. Oxford, UK: Oxford University Press.

Lee, D., Duesbery, L., Han, P., Tashi, T., Her, C., and Ooka Pang, V. (2017). Academic needs and family factors in the education of southeast Asian American students: Dismantling the model minority myth. Journal of Southeast Asian American Education and Advancement, 12(2), 1-28.

Lee, G. L., \& Manning, M. L. (2001). Working with Asian parents and families. Multicultural Education, 9, 23-25.

Lee, J. S. (2002). The Korean language in America: The role of cultural identity in heritage language learning. Language, Culture and Curriculum, 15(2), 117-133.

Lee, J. S. (2014). Community support for Korean as a heritage language in the United States. In T. G. Wiley, J. K. Peyton, D. Christian, S. C. K. Moore, \& N. Liu (Eds.), Handbook of heritage, community, and native American languages in the United States: Research, policy, and educational practice (pp. 253-262). New York, NY: Routledge. 
Li, G. F. (2006). Culturally contested pedagogy: Battles of literacy and schooling between mainstream teachers and Asian immigrant parents. Albany, NY: SUNY.

Li, G. F. (2013). Social class, culture, and Asian social positioning: Rethinking education and power in the new millennium. New Waves - Educational Research \& Development, 16(2), $1-9$.

Li, G. F. \& Wang, J. (2012). Diversity among Asian Americans. In J. Banks (Ed.), Encyclopedia of diversity in education (pp. 151-154). Thousand Oaks, CA: Sage.

Li, G. \& Wen, K. (2015). East Asian heritage language education for a plurilingual reality in the United States: Practices, potholes, and possibilities. International Multilingual Research Journal, 9(4), 274-290.

Li, G. F. \& Yang, L. (2015). A multilevel analysis of Asian immigrant children's reading achievement in the early years: Evidence from the ECLS-K data. Frontiers of Education in China, 10(1), 110-131.

Li, G. F., Hinojosa, D., Wexler, L., Bian, Y., \& Matinez, J. (2017). Using multimodal modules to address pre-service teachers' knowledge gap in learning to teach English language learners. Tapestry, 8(1), 1-35.

Lim, S. A. (2010). Ethnic and racial difference in higher education access: effects of family and school resources. (Unpublished doctoral dissertation). University of California, CA.

Liu, L. L., Benner, A. D., Lau, A. S., \& Kim, S. Y. (2009). Mother-adolescent language proficiency and adolescent academic and emotional adjustment among Chinese American families. Journal of Youth and Adolescence, 38(4), 572-586.

Long, B. T. \& Riley, E. (2007). Financial aid: A broken bridge to college access? Harvard Educational Review, 77(1), 39-63.

Lopez, G. R., Scribner, J. D., \& Mahitivanichcha, K. (2001). Redefining parental involvement: Lessons from high-performing migrant-impacted schools. American Educational Research Journal, 38, 253-288.

Lucas, T., \& Villegas, A. M. (2013). Preparing linguistically responsive teachers: Laying the foundation in preservice teacher education. Theory into Practice, 52(2), 98-109.

Jung, E., \& Zhang, Y. (2016). Parental involvement, children's aspirations, and achievement in new immigrant families. Journal of Educational Research, 109(4), 333-350.

Mehan, H., Hubbard, L., Lintz, A., \& Villanueva, I. (1996). Constructing school success: The consequences of untracking low-achieving students. New York: Cambridge University Press.

Menken, K. (2010). NCLB and English language learners: Challenges and consequences. Theory into Practice, 49(2), 121-128.

Meza, M. (2011). Parental support of Latinos in higher education. (Unpublished doctoral dissertation). University of San Diego, San Diego, CA.

Musu-Gillette, L., Robinson, J., McFarland, J., KewalRamani, A., Zhang, A., \& WilkinsonFlicker, S. (2016). Status and trends in the education of racial and ethnic groups, 2016 (NCES, 2016-007). US Department of Education, National Center for Education Statistics. Washington, DC. Retrieved from http://nces.ed.gov/pubsearch

National Center for Educational Statistics [NCES]. (2007). Education Longitudinal Study of 2002 (ELS:2002): Base-year to second follow-up data file.

National Center for Education Statistics [NCES]. (2015a). The condition of education, 2015 (NCES, 2015-144). Retrieved from https://nces.ed.gov/pubs2015/2015144.pdf 
National Center for Education Statistics [NCES]. (2015b). English language learners. Retrieved October 23, 2018, from https://nces.ed.gov/fastfacts/display.asp?id=96

Paik, S., Kula, S., Saito, L., Rahman, Z., \& Witenstein, M. (2014). Historical perspectives on diverse Asian American communities: Immigration, incorporation, and education. Teachers College Record, 116(8), 1-45.

Paik, S., Rahman, Z., Kula, S., Saito, E., \& Witenstein, M. (2017). Diverse Asian American families and communities: Culture, structure, and education (Part 1: Why They Differ). School Community Journal, 27(2), 35-66.

Perna, L. W. (2000). Differences in the decision to attend college among African Americans, Hispanics, and Whites. Journal of Higher Education, 71, 117-141.

Perna, L. W. (2002). Pre-college outreach programs: Characteristics of programs serving historically underrepresented groups of students. Journal of College Student Development, 43, 64-83.

Perna, L. W. (2007). The sources of racial-ethnic group differences in college enrollment: A critical Examination. New directions for Institutional Research, no. 133. New York, NY: Wiley \& Sons.

Perna, L., \& Titus, M. A. (2005). The relationship between parental involvement as social capital and college enrollment: An examination of racial/ethnic group differences. Journal of Higher Education, 76(5), 485-518.

Pew Research Center. (2012). The rise of Asian Americans. Washington, DC.

Ponitz, C. C., Rimm-Kaufman, S. E., \& Brock, L. L. (2009). Early adjustment, gender differences, and classroom organizational climate in first grade. The Elementary School Journal, 110(2), 142-162.

Portes, A., \& Rumbaut, R. G. (2006). Immigrant America: A portrait. Berkley, CA: University of California Press.

Programme for International Students Assessment [PISA] (2018). PISA 2015 results in focus. Retrieved from https://www.oecd.org/pisa/pisa-2015-results-in-focus.pdf

Raudenbush, S. W. \& Bryk, A. S. (2002). Hierarchical linear models: Applications and data analysis methods. Newbury Park: Sage Publications.

Seal, K. (2010, December 13). Asian-American parenting and academic success. Pacific Standard. Retrieved from https://psmag.com/education/asian-american-parenting-andacademic-success-26053

Sheng, Z., Sheng, Y., \& Anderson, C. J. (2011). Dropping out of school among ELL students: Implications to schools and teacher education. The Clearing House, 84(3), 98-103.

Shin, H. J. (2009). Parental involvement of Asian American immigrant mothers: Investigating social capital, English proficiency, length of US residency, and social class (Unpublished doctoral dissertation). University of Maryland, MA.

Suárez-Orozco, C., Pimentel, A., \& Martin, M. (2009). The significance of relationships: Academic engagement and achievement among newcomer immigrant youth. Teachers College Record, 111(3), 712-749.

Sullivan, A. L. (2011). Disproportionality in special education identification and placement of English language learners. Exceptional Children, 77(3), 317-334.

Sy, S. R. (2006). Rethinking parent involvement during the transition to school: A focus on Asian American families. The School Community Journal, 16, 107-125.

Tabachnick, B. G., \& Fidell, L. S. (2006). Using Multivariate Statistics (5th ed.). Boston, MA: Allyn \& Bacon. 
Teranishi, R. T. (2010). Asians in the ivory tower: Dilemmas of racial inequality in American higher education. New York, NY: Teachers College Press.

Tuan, M. (1998). Forever foreigners or honorary Whites: The Asian ethnic experience today. New Brunswick, NJ: Rutgers University Press.

US Census Bureau. (2010). 2010 census data results for the Asian population and Native Hawaiian and other Pacific Islander population. Retrieved from http://www.apiidv.org/files/2010Census-WHIAAPI-2011.pdf

Uy, P. S. (2015). Supporting southeast Asian American family and community engagement for educational success. Journal of Southeast Asian American Education and Advancement, 10(2), 1-14.

Vygotsky, L. S. (1978). Mind in society. Cambridge, MA: Harvard University Press.

Vygotsky, L. S. (1987). The collected works of L. S. Vygotsky (R. W. Rieber \& A. S. Carton, Trans.). New York, NY: Plenum.

Woo, H. J. (2009). Investigation on multiple factors affecting English-language learners' reading achievement: Hierarchical linear modeling approach. (Unpublished doctoral dissertation). University of Illinois at Urbana-Champaign, IL.

Yeh, E. (2014). Parental involvement in non-native English speakers' postsecondary enrollment (Unpublished doctoral dissertation). Ohio University, Athens, Ohio.

Zhao, Y. (2011). Handbook of Asian education: A cultural perspective. Lei, J., Li, G., He, M. F., Okano, K., Megahed, N., Gamage D., \& Ramanathan, H. (Eds.). New York, NY: Routledge Taylor and Francis Group. 


\section{Appendix}

Table A: Composite and Index Variable Descriptions (Adopted from Yeh, 2014) Variable name Labels and values

Parental Involvement

Parent-School Involvement

Parent reports contacting the student's school about doing volunteer work.

Parent contacted the school about NNESs' academic performance

Parent contacted school about plans after high school

Parent contacted the school about

NNESs' course selection

Students have problematic behavior at school
BYP53H: Parent contacted school about fundraising/volunteer work (1-4) ( $1=$ None; $4=$ More than four times)

BYP53B Parent contacted school about school program for year $(1-4)$ $(1=$ None; $2=$ Once or twice; $3=$ Three or four times; $4=$ More than four times)

BYP53C: Parent contacted school about plans after high school (1-4)

BYP53D: Parent contacted school about course selection $(1-4)$

BYP53F: Parent contacted school about problem behavior (1-4)

\section{Parent-Student Involvement}

Parent-student discussions about education-related issues, and parent's advice for NNESs' school work
BYS86A: How often discussed school courses with parents $(1-3)$

BYS86C: How often discuss things studied in class with parents $(1-3)$

BYS86D: How often discussed grades with parents $(1-3)$.

BYS86F: How often discussed prep for ACT/SAT with parents (1-3)

BYS86G: How often discussed going to college with parents $(1-3)$

(1=Never; $2=$ Sometimes; $3=$ Often $)$ 
Parent's educational expectations

Parent's values/ norms toward education: Parent has rules about GPA.
BYS65A: How far in school mother wants 10 th grader to go $(1-7)$

BYS65B: How far in school father wants 10th grader to go $(1-7)$. ( $1=$ Less than high school graduation; $2=$ High school graduation or GED only; $3=$ Attend or complete 2-year college/school; 4=Attend college, 4-year degree incomplete; $5=$ Graduate from college; $6=$ Obtain Master's degree or equivalent; $7=$ Obtain $\mathrm{PhD}, \mathrm{MD}$, or other advanced degree).

BYP69A: Family rules for 10th grader about maintaining grade average $(0=$ No; $1=$ Yes $)$.

Home Language Environment

NNESs' frequency of native language use

Parent speaks language other than English at home
BYS69A: How often 10th grader speaks native language with mother (1-4)

BYS69B: How often 10th grader speaks native language with father. (1=Never; 2 =Sometimes; $3=$ About half of the time; $4=$ Always or most of time).

BYP30B: How often parent speaks native language with children (1-4)

\section{Covariates: SES Factors}

Mean Income

$$
\begin{aligned}
& \text { ALLSCHOOLSES: Mean total family } \\
& \text { income from all sources, } 2001-\text { composite. } \\
& (1=\text { None; } 2=\$ 1,000 \text { or less; } 3=\$ 1,001- \\
& \$ 5,000 ; 4=\$ 5,001-\$ 10,000 ; 5=\$ 10,001- \\
& \$ 15,000 ; 6=\$ 15,001-\$ 20,000 ; 7=\$ 20,001- \\
& \$ 25,000 ; 8=\$ 25,001-\$ 35,000 ; 9=\$ 35,001- \\
& \$ 50,000 ; 10=\$ 50,001-\$ 75,000 ; \\
& 11=\$ 75,001-\$ 100,000 ; 12=\$ 100,001- \\
& \$ 200,000 ; 13=\$ 200,001 \text { or more })
\end{aligned}
$$


Family Income

Parents' Level of Education
BYP85: Total family income from all sources, 2001(1-13)

BYP34A: Parents' highest level of education completed (1-8) (1=Did not finish high school; 2=Graduated from high school or GED; $3=$ Attended 2-year school, no degree; 4=Graduated from 2-year school; 5=Attended college, no 4-year degree; $6=$ Graduated from college; $7=$ Completed Master's degree or equivalent; $8=$ Completed $\mathrm{PhD}, \mathrm{MD}$, advanced degree).

\section{Covariates: Linguistic Factors}

Reading test standardized score (IRT)

NNESs' self-reported English language ability
BYTXRSTD: Reading test standardized score (1-100) (Min:22.57; Max: 78.76; Reading standardized T Score)

BYS70A: How well 10th grader understands spoken English (1-4)

BYS70B: How well 10th grader speaks English (1-4)

BYS70C: How well 10th grader reads English (1-4)

BYS70D: How well 10th grader writes English (1-4)

$(1=$ Very well; $2=$ Well; $3=$ Not well; $4=$ Not at all).

i Race/ethnicity is reported as follows: Black includes African American, Hispanic includes Latino, Asian includes Native Hawaiian or other Pacific Islander, and American Indian includes Alaska Native. "White" is predominantly White, with a very small number of individuals from other race categories. All race categories exclude individuals of Hispanic or Latino origin (NCES, 2007, p. 9).

ii The study included all the regression coefficients for the final model of Asian group NNESs and showed all the predictors at the final step (see Appendix A). 